\title{
Il saggio come concezione metafisica in Leonardo Sciascia
}

\author{
Samir Ben Ahmed
}

Dopo aver puntualizzato sociologicamente il fenomeno della mafia attraverso le pagine de Le parrocchie di Regalpetra, e una volta superata l'esigenza prettamente saggistica, Sciascia pubblica nel 1961 un'opera intitolata Il giorno della civetta. ${ }^{1}$ In essa l'autore approda a una definitiva maturazione letteraria che gli permette di armonizzare le strutture del racconto e la "materia saggistica".

Su uno sfondo mafioso Leonardo Sciascia così orchestra il suo racconto: Salvatore Colasberna viene ucciso a colpi di lupara e con lui scompare un onest'uomo: Paolo Nicolosi. Nella zona si trova da poco tempo un capitano dei carabinieri settentrionale che ha fatto la Resistenza. Da ex partigiano ha una visione precisa della vita e della sua missione; cerca nelle sue indagini di non lasciarsi fuorviare da nulla e da nessuno. Organizzata una rete di informatori, fa arrestare diversi mafiosi di secondo piano. Il suo intento tuttavia è di arrivare fino ad un capomafia: don Mariano Arena. Nel momento in cui il suo scopo è quasi raggiunto, entra in scena la macchina delle clientele e l'inesperto capitano, recatosi a Parma per una breve licenza, al suo ritorno trova alibi prefabbricati per tutti gli incriminati, che escono trionfanti dal carcere; al capitano Bellodi non resta che tornarsene a Parma e meditare su quello che è la giustizia. Ora, benché i personaggi principali del romanzo siano due, don Mariano Arena e il capitano Bellodi, il vero protagonista è l'universo insulare e le sue sotterranee, travolgenti forze sovversive.

La domanda di base che ci facciamo è: perché Sciascia sente il bisogno di servirsi della struttura schematica del romanzo poliziesco, sconvolgendola allo stesso tempo?

La scelta del genere poliziesco da parte di Sciascia ha suscitato l'interesse di molti studiosi, ${ }^{2}$ che, da un lato, hanno focalizzato l'attenzione sulle deviazioni strutturali rispetto al romanzo giallo classico e, dall'altro, hanno messo in evidenza i temi socio-politici immessi nella trama poliziesca. Già nel 1974, Claude Ambroise poteva scrivere:

La forma del giallo in quanto prodotto ideologico ha evidentemente un significato politico. Sempre. Ma nei libri di Sciascia, c’è qualcosa di più e cioè una diretta immissione della politica nella trama romanzesca: [ . . ] si parte dalla cronaca nera per approdare alle sfere politiche. Questo movimento è manifesto ne $I l$ giorno della civetta, diventa più sottile e acquista maggiore ampiezza nelle opere successive. (141) 
Per il critico francese, infatti, "il giallo diventa la forma peculiare della riflessione sul sistema politico" (ibid.); con l'ovvia differenza rispetto al giallo anglosassone che la verità dei fatti non viene scoperta né vengono puniti $\mathrm{i}$ colpevoli. L'incapacità dell'investigatore sciasciano di scoprire la verità ne fa, secondo Giovanna Jackson, un anti-eroe. Per la Jackson, infatti:

The detectives of Sciascia are gifted with all the abilities of a Dupin or a Sherlock Holmes, yet they consistently fail to bring order to chaos and to re-establish the balance that the crime had destroyed. (12)

La studiosa ha identificato così una caratteristica costante comune a tutti gli investigatori sciasciani. Il motivo che essa propone per questa impossibilità è l'inadeguatezza della ragione - la base sulla quale riposa il giallo classico nell'ambiente socio-politico in cui opera l'investigatore. ${ }^{3}$

Il leitmotiv, che attraversa tutti i contributi della critica intorno al $G C$, è che ci si trova davanti a un saggio-denuncia della mafia siciliana presentato nella forma di un giallo. Per dirla con le parole di Luigi Cattanei:

Il ricorso al genere "giallo" non si deve solo alla tecnica narrativa che "impedisce al lettore di lasciare a metà il libro", ma all'intento di denuncia che Sciascia maturò riflettendo sul fenomeno mafioso. Il giallo tradizionale s'anima in lui d'una personale vibrazione agli eventi, la Sicilia di oggi esclude ogni altra scelta spaziotemporale e carica il "caso" di significati etico-politici, talché l'autore può trascurare l'esito dell'indagine intrecciata a un gioco politico trasparente e pur agilmente svincolato dai modi del saggio. (58)

Oltre alla questione etico-politica, Nicola Fano introduce il tema della morte, "intesa come spinta che conduce l'uomo a scegliere di dar morte a un suo simile", e "l'intento etico e sociale [dell'autore] di indicare i limiti possibili della vocazione mortifera dell'vomo e, eventualmente, di porre un freno ad essa" (35). Per Fano le due chiavi di lettura del $G C$ sono, in effetti, l'impegno etico-politico dell'autore e la "vocazione mortifera" dell'uomo:

Appare immediatamente chiaro, dunque, che le chiavi di lettura del Giorno della civetta sono sostanzialmente due: una di ordine politico, legata ai rapporti oscuri fra la gestione del potere pubblico e quella del potere mafioso; l'altra di ordine morale, relativa a ciò che abbiamo chiamato vocazione mortifera dell'essere umano. Ed è altrettanto chiaro che la terra di Sicilia, con la sua specialissima commistione fra ragione e mafia, fra utopia e disincanto (che affonda profonde radici nell'antropologia del suo popolo) offre a Sciascia un terreno di studio ineguagliabile. Proprio la Sicilia [. . .] costituisce il terzo territorio di sviluppo del romanzo; ma si tratta di una "zona franca", di un territorio onnicomprensivo all'interno del quale le due precedenti chiavi di lettura convivono e interagiscono. (37)

La scelta del modello poliziesco, in effetti, porge alla scrittura di Sciascia due vantaggi importanti. Un tale genere preconizza intanto l'esistenza di una ragione 
superiore - che Sciascia chiama metafisica - la quale mette in rilievo la "positività" dell'investigatore rispetto alla "negatività" del criminale. In piu, questo genere esime lo scrittore dalla piatta scrittura sociologica del saggio moralizzante e fornisce una struttura narrativa che dà al discorso maggiore forza di convincimento tramite la contrapposizione drammatica di due mondi: quello oscuro della mafia e quello della "Grazia illuminante" della ragione.

In queste pagine, si cercherà di dimostrare come Sciascia ricorra a certe caratteristiche della scrittura poliziesca nella stesura del saggio - inteso come éssai -, ossia come il giallo assuma una funzione strutturale nell'elaborazione della tesi di fondo. Nel caso dei romanzi polizieschi di Sciascia e in particolar modo del $G C$, il primo romanzo di questo genere, sarebbe più opportuno parlare di romanzi a tesi scritti da uno scrittore di parte.

Il titolo, Il giorno della civetta, è un ossimoro che richiama l'attenzione su due mondi contrapposti: il giorno, che sta per la luce della ragione, e la civetta, uccello notturno, che sta per l'oscurità della mentalità mafiosa. Sin dall'inizio il narratore ci pone in presenza del mondo "oscuro" del romanzo. All'apertura del racconto prevale un'atmosfera cupa di paura e di omertà, ove la reticenza dei testimoni oculari nel partecipare alla ricostruzione dell' assassinio, deriva dalla forte presenza della mafia con la sua sbrigativa giustizia, che non ammette interferenze e che riesce ad imporsi per mezzo della intimidazione. ${ }^{4}$ I due colpi di lupara sono un chiaro avvertimento per chi si trova nelle vicinanze: chi ha visto non deve parlare, pena la morte. Si tratta di una mentalità che è "la forma più netta della costituzione siciliana, del modo di essere siciliano e della legge non scritta che ne scaturisce, [e che] si riduce a un'intesa di persone dei medesimi pensamenti, del medesimo sentire"; (Sciascia, La mafia 82) di un modo irrazionale agli occhi di chi rappresenta la giustizia dello stato.

$\grave{E}$ interessante osservare che in questo primo romanzo l'impalcatura del giallo è ben più riconoscibile che non in quelli successivi. Il romanzo si apre, appunto, con il morto ammazzato e le circostanze del delitto sono rivelate parzialmente al lettore, senza cioè rivelare immediatamente l'identità dell'omicida, né il movente. Di qui scatta l'indagine con le tipiche tecniche investigative, con la raccolta, cioè, di indizi che poi diventano prove e di interrogatori che diventano confessioni. $\mathrm{Ma}$ se nel giallo tradizionale si assiste allo scontro fra due individui, l'investigatore e l'assassino, con la messa a fuoco della superiorità intellettuale del primo, ${ }^{5}$ nel $G C$ si ha invece uno scontro fra due mondi, ciascuno con la propria filosofia chiaramente definita: il mondo dei mafiosi siciliani e dei loro fiancheggiatori ai posti chiave del potere da un lato, e dall'altro Bellodi - "che riteneva la legge scaturita dall'idea di giustizia e alla giustizia congiunto ogni atto che la legge muovesse" (408) - , solo contro tutti, con il suo mondo ideale di ragione, di giustizia uguale per tutti e di verità univoca. C'è, infatti, nella figura di Bellodi, un motivo di fondo che ne costituisce la cifra: è il forestiero, il partigiano settentrionale affascinante e temibile per la gente del paese. Affascinante per la sua cortesia e le sue maniere rispettose dell'individuo (anche se criminale), temibile 
perché detiene un potere. In fondo il lettore lo percepisce come uno straniero alle prese con una cultura radicalmente diversa dalla sua, ${ }^{6}$ di cui intuisce le ragioni storiche, ma contro cui ha dichiarato guerra.

$\mathrm{Da}$ un punto di vista strettamente strutturale, le indagini sono un pretesto per l'esposizione di queste due filosofie e per l'individuazione di quella zona franca, "terra di nessuno" che alternativamente le separa e le unisce: una "terra di nessuno" riconoscibile nella dimensione ove si aggira Parineddu, il confidente, che giocando d'azzardo rischia la vita tra mafia e carabinieri. ${ }^{7}$ Così l'opposizione tra don Mariano e il capitano Bellodi si configura come una guerra nel corso della quale assistiamo ad un emblematico "saluto delle armi" scambiato fra i due maggiori contendenti:

Lei, anche se mi inchioderà su queste carte come un cristo, lei è un uomo . . . "Anche lei" disse il capitano con una certa emozione. E nel disagio che subito senti di quel saluto delle armi scambiato con un capo mafia, a giustificazione pensò di avere stretto le mani, [. . .] al ministro Mancuso e all'onorevole Livigni: sui quali don Mariano aveva il vantaggio di essere un uomo. (407)

Questa contrapposizione è importante per l'autore ai fini dell'esposizione della sua tesi di fondo che regge tutto il romanzo. Intanto, dal passo appena citato, si ricava più chiaramente la posizione di Bellodi: egli non appartiene al mondo siciliano, benché comprenda le ragioni storiche che lo hanno modellato, né si riconosce nel potere centrale, ancorché ne sia il rappresentante e il difensore delle leggi. Come si vedrà in seguito, è in mezzo a questi opposti grazie ai quali Sciascia riesce a guidare il lettore verso la conclusione finale: la visione mafioso-siciliana delle cose si è ormai estesa al resto dell'Italia. In tal modo l'autore riesce a veicolare una "lettura" personalissima e soggettiva di certi aspetti dei rapporti tra la Sicilia e il continente.

Come hanno già osservato molti critici, il $G C$ nasce dunque dalla fusione di due generi letterari: il saggio, per la materia, ed il "giallo", per la tecnica narrativa. ${ }^{8} \mathrm{Ma}$ è importante osservare che entrambi i generi, pur mantenendo la propria autonomia stilistica, si avvalgono del processo "argomentativo" tipico dell'esposizione dimostrativo-deduttiva. Vale a dire che la questione di fondo la 'colpa' nel giallo e la 'tesi' nel saggio - deve far capo a un ragionamento logico, nel quale due fattori fondamentali (verità e impostura, innocenza e colpevolezza, bene e male, opinione propria e quella corrente) si combinano e si contrappongono. Ciò che prevale e determina il successo del ragionamento, e in ultima analisi attira di più l'attenzione, è il procedimento analitico: la conclusione diventa così inconfutabile e, soprattutto, l'unica possibile. A proposito della "fusione" dei due generi, Sciascia sostiene che la sua

è una materia saggistica che assume i modi del racconto, si fa racconto. Il processo di trasformazione non eे facile: e percid io sono particolarmente attento e accorto nella tecnica del raccontare. Spesso anzi mi servo della tecnica in un certo senso più sleale nei riguardi del lettore, quella che impedisce al lettore di lasciare a metà 
un libro; la tecnica, voglio dire, del romanzo poliziesco. Con questa formula mi sono piuttosto garantito rispetto alla crisi della narrativa. Ammesso che la narrativa sia in crisi. (Mauro 2)

In base a ciò che si è già detto, la "slealtà" di questa tecnica non scaturisce solamente dall'imposizione dell'obbligo della lettura (che è pur sempre una scelta), bensì da una tutt'altra costrizione ben più sottile e vincolante, che è quella che più importa in un contesto ove è vitale convincere: l'obbligo di condividere le opinioni dell'autore che sorreggono e compongono l'impianto narrativo. La struttura e la tecnica del giallo forniscono, oltre ai "modi", il mezzo per guidare le idee e le prese di posizione dell'autore; così, per dirla con Sciascia, la "materia saggistica ... si fa racconto". Proprio a questa "trasformazione" (o travestimento) della materia, che è una realtà socialmente e storicamente localizzabile, è finalizzato lo stile che tende alla creazione di un senso delle cose siciliane ma che, al contempo, giustifica e condanna il "comune sentire" tipico della mentalità mafioso-siciliana. Il ricorso al proverbio e al soprannome, con il loro valore denotativo e connotativo, ${ }^{9}$ svolge la stessa funzione del richiamo all'opera di autori siciliani nella descrizione di un volto o di un atteggiamento:

Il vecchio disse che forse il nome giusto era Barricieddu, o forse Bargieddu: ma in ogni caso significava malvagità, la malvagità di uno che comanda; che un tempo i Barruggieddi o Bargieddi comandavano i paesi e mandavano gente alla forca, per piacere malvagio .... (455)

In primo luogo, si osservi il grado di puntiglio linguistico-lessicale, del tutto incongruo nel discorso di un pastore siciliano. Eे chiaro che è l'autore ad insistere su un dettaglio "saggistico" e con tale insistenza ne fa, appunto, "racconto" e così crea quello che ho chiamato "il senso delle cose siciliane". Subito dopo il narratore presenta la conclusione o, meglio, l'uso che il lettore deve fare di tale informazione. Un uso che serve non tanto al giallo quanto al saggio. Infatti, la conclusione è doppia: a) è inutile insistere in questo interrogatorio e b) la Sicilia e i siciliani non si fidano di altra legge che della loro propria:

Ma capì che non c'era niente da cavare da uno che riteneva il capo degli sbirri cattivo quanto il proprio cane. E non è che avesse torto, pensava il capitano: da secoli i bargelli mordevano gli uomini come lui, magari li facevano assicurare, come diceva il vecchio, e poi mordevano. Che cosa erano stati i bargelli se non strumenti dell'usurpazione e dell' arbitrio? (455)

Queste considerazioni non assolvono ad alcuna funzione nella scrittura poliziesca, dove tutto dev'essere finalizzato, programmaticamente inteso a nascondere o a evidenziare una pista o un indizio che ritornerà al momento del finale riassemblaggio del casse-tête. Lo stesso dicasi per le divagazioni liriche che ricorrono spesso in questa prosa e per certe descrizioni paesaggistiche che non contribuiscono affatto all'intreccio, bensì a comporre quel tessuto puramente narrativo che lega la "materia" saggistica ai "modi del racconto". Pertanto la particolarità di 
questo primo giallo sciasciano va rintracciata non solo nella nuova scrittura che nasce dalla fusione dei due generi, ma anche e soprattutto nell'impatto che essa ha sul lettore.

Presi separatamente i due generi presentano due posizioni diverse dell'autore e percio due altrettanto diverse aspettative del lettore. Il saggio, qui inteso nella sua accezione settecentesca di éssai e di pamphlet, è tradizionalmente in stretto rapporto con la filosofia e la visione del mondo del saggista; prospettive esposte in modo da ricondurre il lettore all'autore e alla sua posizione etico-politica, in cui il lettore è libero di mantenere una distanza critica. Cid gli fornisce un minimo di respiro, permettendogli di accettare o di rifiutare integralmente o in parte le idee dell'autore.

La scrittura giallistica, al contrario, è per definizione coinvolgente, perché l'autore nel districare l'enigma si appella ad una logica "metafisica" ritenuta comune a tutti; e la distinzione che fa tra bene e male non ammette equivoci. A questo riguardo Sciascia scrive:

Nella sua forma più originale ed autonoma, il romanzo poliziesco presuppone una metafisica: l'esistenza di un mondo "al di là del físico", di Dio, della grazia - e di quella grazia che i teologi chiamano illuminante. Della Grazia illuminante l'investigatore si può anzi considerare il portatore [. . .] . L'incorruttibilità e infallibilità dell'investigatore, la sua quasi ascetica vita [. . .], il fatto che non rappresenta la legge ufficiale ma la legge in assoluto, la sua capacità di leggere il delitto nel cuore umano oltre che nelle cose, ciò negli indizi, e di presentirlo, lo investono di luce metafisica, ne fanno un eletto. ("Breve storia" 218)

Queste qualità inducono chi legge ad immedesimarsi nell'investigatore e quindi a condividere la "filosofia" e le conclusioni dell'autore, proprio perché non sono più ritenute estranee alle proprie.

Combinando i due generi, Sciascia ottiene un saggio scritto in margine al giallo. In altre parole, egli sostituisce alla visione del mondo universale e "oggettiva" del giallo classico quella personale e soggettiva del saggio. ${ }^{10} \mathrm{La}$ "slealtà" nei confronti del lettore, di cui si è detto poc'anzi, scaturisce proprio dal fatto che le indagini e la scoperta per gradi dei fatti mettono in moto un sottile meccanismo di convincimento. Tale meccanismo porta il lettore, "costringendolo", a condividere inconsciamente la presa di posizione dell'autore, vale a dire: la Sicilia, scaturigine della non-ragione, del sopruso, del malcostume etico-politico ecc., ha esteso i suoi influssi nefasti al di là dello stretto di Messina:

Bellodi disse che la Sicilia era incredibile. "Eh sì, dici bene: incredibile . . . Ho conosciuto anch'io dei siciliani ... E ora hanno la loro autonomia, il loro governo ... il governo della lupara, dico io . . . incredibile: è la parola che ci vuole". Incredibile è anche 1'Italia: e bisogna andare in Sicilia per constatare quanto è incredibile l'Italia. Forse tutta l'Italia va diventando Sicilia . . . A me è venuta una fantasia, leggendo sui giornali gli scandali di quel governo regionale: gli scienziati dicono che la linea della palma, cioe il clima che è propizio alla vegetazione della palma, 
viene su, verso il nord, di cinquecento metri, mi pare, ogni anno . . . La linea della palma ... Io invece dico: la linea del caffe ristretto, del caffè concentrato . . . E sale come l'ago di mercurio di un termometro, questa linea della palma, del caffe forte, degli scandali: su su per l'Italia, ed è oltre Roma . . . ". (479)

Poiche la punizione di don Mariano è stata impedita, questa, che è la conclusione del saggio, è in sostanza anche la conclusione del romanzo. In effetti, date le forze contrapposte messe in moto nel romanzo, è prevedibile che la spinta narrativa non tenda verso la soluzione consolatoria del caso e la punizione del colpevole, ma verso la dimostrazione di come il colpevole non possa essere punito. Lo Stato, infatti, l'unico abilitato a farlo, si è lasciato contaminare dalla rampante "pazzia" della Sicilia nella persona di coloro che dovrebbero vegliare a che la sua giustizia non venga soppiantata da nessun'altra forma sostitutiva: nel caso particolare dalla giustizia della lupara. A lettura compiuta, nella memoria del lettore non rimangono tanto i personaggi e le situazioni, quanto l'accettazione della tesi di fondo, cioè che "la linea della palma sale . . . su su per l'Italia ed è oltre Roma". E da ciò deriva un senso di disagio perché a chi ha seguito la storia rimane solo da sottoscrivere al mondo "eletto", il cui recupero l'autore propone implicitamente: il mondo della "grazia illuminante" della ragione, quello ideale che vagheggia Bellodi, insomma.

Nel lettore del giallo classico vi è, inoltre, un senso di appartenenza ad un mondo univoco in cui la distinzione fra il bene il male è chiaramente delineata. Un mondo di cui l'autore, con la punizione del colpevole, asserisce la legittimità e l'univocità. Nel GC Sciascia impedisce al lettore di sentirsi parte tanto del mondo siciliano quanto di quello continentale; l'unica alternativa rimasta è di accettare, a costo di rompersi la testa, ${ }^{11}$ la tesi di fondo del romanzo e la visione che la sorregge, corroborando così la soluzione suggerita da Bellodi:

"Questo è il punto" pensd il capitano "su cui bisognerebbe far leva. [. . .] Qui bisognerebbe sorprendere la gente nel covo dell'inadempienza fiscale, come in America. [. . . E tutte quelle volpi, vecchie e nuove, che stanno a sprecare il loro fiuto dietro le idee politiche o le tendenze o gli incontri dei membri più inquieti di quella grande famiglia che è il regime, e dietro i vicini di casa della famiglia, e dietro i nemici della famiglia, sarebbe meglio si mettessero ad annusare intorno alle ville, le automobili fuori serie, le mogli, le amanti di certi funzionari: e confrontare quei segni di ricchezza agli stipendi, e tirarne il giusto senso. Soltanto così ad uomini come don Mariano comincerebbe a mancare il terreno sotto i piedi ... . In ogni altro paese del mondo una evasione fiscale come quella che sto costatando sarebbe duramente punita: qui don Mariano se ne ride, sa che non gli ci vorrà molto per imbrogliare le carte". (465-66)

In questo lungo monologo interiore, il più rappresentativo fra $\mathrm{i}$ tanti disseminati nel testo, è chiaro che Bellodi funge da portavoce dell'autore e che queste considerazioni non fanno parte della tessitura logica e percio non avrebbero alcuna funzione in un romanzo poliziesco tradizionale, che mira esclusivamente a far 
collimare $\mathrm{i}$ fatti e a seguire un ragionamento puramente razionale. Ma sul versante saggistico queste considerazioni espletano una funzione notevole: forniscono, intanto, un ulteriore motivo della sconfitta di Bellodi e rappresentano la strada da battere, suggerita dall'autore, per sconfiggere la mafia e ciò che essa rappresenta.

Il rapporto dialettico tra saggio e romanzo poliziesco, che abbiamo delineato, viene suffragato, e le eventuali falde cauterizzate da una coscienza pirandelliana della verità proprio nel momento in cui don Mariano capitola: "La verità è nel fondo di un pozzo: lei guarda in un pozzo e vede il sole o la luna; ma se si butta giù non c'è più né sole né luna, c'è la verità (469).

Così Sciascia per bocca di don Mariano anticipa la fine dell'interrogatorio e dunque la fine della trama poliziesca, perché l'inchiesta finisce. A questo punto, continuando la metafora pirandelliana di don Mariano, Bellodi ha visto la verità nel pozzo della Sicilia. Ma, dalle pagine che seguono, il lettore capisce che Bellodi, pur avendo raggiunto "il fondo del pozzo" e trovato la verità, non sarà capace di riportarla alla superficie, di presentarla come la Verità. Bellodi chiude il romanzo con quel "mi ci romperò la testa", che ci riconduce alla metafora di don Mariano.

Nel $G C$, Sciascia ha assunto una situazione storica che è ben definita e già nota ai suoi lettori italiani. In ultima analisi la "materia" saggistica sta nella scelta di questa situazione che era (ed è) quotidianamente esposta nella cosiddetta cronaca nera dei giornali e delle riviste italiane e che richiede anche una certa verosimiglianza nell'agnizione finale: la mafia non può essere sconfitta, perché i fatti dimostrano che non lo è mai stata. Ogni altra conclusione, oltre che smisuratamente ottimistica, sarebbe inaccettabile, perché non reggerebbe alla prova dei fatti. Ma la ragione della non-sconfitta della mafia, che Sciascia avanza - e qui sta la conclusione verso la quale tende la spinta saggistica e di conseguenza tutto il romanzo — è che lo stato si è "sicilianizzato" ed è divenuto corrotto, perdendo il vero senso della giustizia.

\section{McGill University}

\section{NOTE}

1 D'ora in avanti $G C$.

2 Per via del gran numero degli interventi su questo particolare punto, ne presenteremo solo quattro tra i più rappresentativi.

3 Cfr. Jackson: "The cool analytical procedures of the investigators crash against the monolythic wall of a criminal society, and their carefully constructed detection ends in a fiasco. At the end of Sciascia's detective novels, the criminals are left unpunished, disorder and uncertainty predominate. Sciascia's representatives of logic, order and ethics are destroyed by the collective criminal society. Their own inability to conform and to make the necessary compromises for survival is their "tragic flaw' which leads them to their downfall." (12)

4 A questo proposito, sarà utile ricordare le parole dell'antropologo Anton Block secondo cui "certe espressioni del linguaggio quotidiano mostrano il ruolo occupato dalla violenza 
illegale nella società contadina della Sicilia. I mafiosi erano definiti uomini 'd'onore', 'di rispetto', 'd'autorità'; sapevano 'badare ai fatti propri' e 'farsi rispettare'. La questione centrale è l'esistenza di un codice di comportamento che si riassume nel termine 'omertà'. Secondo questo codice, un uomo si fa rispettare mantenendo il silenzio sui 'crimini' di cui è stato testimone, vittima o esecutore. Reticenza e segretezza, a volte vere e proprie cospirazioni del silenzio specialmente di fronte all'autorita giudiziarie, erano l'espressione dell'autorità e dell'influenza dei tradizionali àmbiti di potere locali. Al debole era imposto di tacere: 'cantare' implicava fare appello a risorse esterne che avrebbero minacciato gli equilibri tradizionali. Attraverso la manipolazione di questo complesso codice culturale e del controllo sociale che esso sottintendeva, i mafiosi cercavano di tener lontana la popolazione locale dalla concorrenza dei poteri esterni. La gente era costretta ad appoggiarsi a loro: ignorare il codice equivaleva a un tentativo di scavalcare il mediatore del potere. Sebbene in misura diversa, l'omertà pervadeva l'intera struttura della comunità". (16)

5 Per maggion dettagli sulle caratteristiche strutturali del romanzo poliziesco rimandiamo in particolar modo al romanzo L'ambulante, di Peter Handke e allo studio compiutone da Victor Zmegac: "Aspekte des Detectivromans", ora in La trama del delitto.

6 Cfr. Jackson: "Bellodi is a stranger in two ways: first, and most obviously, he is a man from the North and almost a foreigner even to the local idiom of the small Sicilian town where he is to spend some time as the police captain. [Secondly] Bellodi's own morality and belief in justice do not permit him to consider compromises, nor to accept secrets, nor even to understand the sacredness of silence among Sicilians"(14).

7 "Ma tra mafia e carabinieri, le due parti tra cui si muoveva il suo azzardo, la morte poteva venirgli da una sola parte. Da questa parte non c'era la morte, c'era quest'uomo biondo e ben rasato, elegante nella divisa; quest'uomo che parlava mangiandosi le esse, che non alzava la voce e non gli faceva pesare disprezzo: e pure era la legge, quanto la morte paurosa; non, per il confidente, la legge che nasce dalla ragione ed è ragione, ma la legge di un uomo, che nasce dai pensieri e dagli umori di quest'uomo, [ . . ] l'assoluta irrazionalità della legge, ad ogni momento creata da colui che comanda [. . .] da chi ha la forza, insomma". (407)

8 Cfr. Ambroise; Cattanei; Jackson; Fano. Si veda anche Madrignani che scrive: "[le inchieste di Sciascia] sono avventure intellettuali in cui lo sbocco non consiste nel raggiungere una verità uiltima e definitiva, e così appagare lo stato d'attesa del lettore. Non si tratta di romanzi gialli secondo la consecutio causa-effetto della loro retorica narrativa. Non l'appagamento finale ma la ricerca del percorso è di per sé l'avventura mentale e artistica di cui si sostanzia il 'romanzo' dell'ultimo Sciascia. Gioco d'intelligenza felicemente legato alla logica deduttiva, questa arte ingloba tuttavia, senza rinnegare la propria normativa ludica, un elemento d'impegno civile abilmente mimetizzato, ma decisivo. C'è alle spalle la grande tradizione illuministica francese, con quel tanto di seriamente giocoso che conosciamo, mentre rimane sullo sfondo il gusto dei sapienti meccanismi del giallo [. . . ] con la grande sorpresa finale che spiazza il lettore. Per Sciascia non si tratta di mettere in moto una serie di dubbi fittizi per ingannare e trascinare su false piste il lettore, ma di sollevare dubbi anche là dove non ne esistono, dove non sarebbe economico sollevame". (140)

9 Tra le pagine 417 e 419 è racchiuso un mini-trattato sulle ingiurie (soprannomi) in Sicilia, sul loro valore, e su quanto riescano a cogliere in una parola un tratto fisico o caratteriale che riassume un individuo. Tramite i vari impieghi di un'intera gamma di opinioni, assunti, concetti e preconcetti che hanno accompagnato i siciliani da sempre, Sciascia pừ storicizzare e al contempo rendere atemporali situazioni e fatti di cronaca. La sicilianità, "compiuta forma di realtà umana", viene usata da lui come un canone per giudicare e parametrare il caos e la civiltă, il desiderio e la paura inerenti a tutti gli uomini. Questa Sicilia simbolo della non-ragione e del malcostume politico e morale, è la comice dei romanzi che 
nella struttura si ispirano al genere poliziesco e la cui narrazione, nel corso delle indagini, oscilla tra la critica sociale e le vicende emblematiche del protagonista.

E interessante rilevare che questa "manipolazione" è riscontrabile in tutte le opere di Sciascia, persino quelle ritenute "storiche" (Il consiglio d' Egitto, La morte dell' inquisitore, Atti relativi alla morte di Raymond Roussel, La scomparsa di Majorana, I pugnalatori, L'affaire Moro, La strega e il capitano e Porte aperte). Nel caso di questi romanzi il lettore è di fronte alla sintesi di una ricerca storico-investigativa dell'autore stesso o di un suo personaggio. Nel corso di questa ricerca, il fatto storico viene inserito in una struttura narrativa - simile a quella del giallo - che ne assicura la giusta interpretazione, ciod quella voluta dall'autore secondo lo schema che abbiamo presentato sopra. Sciascia sovverte, infatti, la nozione manzoniana della storia come scheletro (nel suo saggio "Del romanzo storico", Manzoni usa il termine "carcame") e ne fa un elemento attivo che agisce direttamente sui personaggi condizionandone il caraftere e le motivazioni (si pensi all'abate Vella de Il consiglio d'Egitto). La storia diventa così un elemento non più fisso e univoco, bensì personale e, percio, interpretabile. Sicché, tra la versione del libro di storia e quella di un romanzo di Sciascia, il lettore tende a sottoscrivere a quest'ultima.

11 Il romanzo si conclude appunto su questa frase profetica "mi ci rompero la testa".

\section{OPERE CITATE}

Ambroise, Claude. "Giallo e politica". Invito alla lettura di Sciascia. Milano: Mursia, 1974. 141-45. Block, Anton. La mafia di un villaggio siciliano 1860-1960. Imprenditori, contadini, violenti. Torino: Einaudi, 1986.

Cattanei, Luigi. Leonardo Sciascia. Introduzione e guida allo studio dell'opera sciasciana. Storia e antologia della critica. Firenze: Le Monnier, 1979.

Fano, Nicola. Come leggere II giorno della civetta di Leonardo Sciascia. Milano: Mursia, 1993.

Handke, Peter. L' ambulante. Milano: Feltrinelli, 1970.

Jackson, Giovanna. Leonardo Sciascia: 1956-1976. A thematic and structural study. Ravenna: Longo, 1981.

Madrignani, Carlo A. "Il gioco degli enigmi". Leonardo Sciascia. La verità, l'aspra verità. Ed. Antonio Motta. Manduria: Laicata, 1985. 138-46.

Mauro, Walter. Sciascia. Firenze: La Nuova Italia, 1970.

Sciascia, Leonardo. II giorno della civetta. Opere. Vol. 1. Milano: Bompiani, 1987. 387-483. . La mafia. Bologns: Boni, 1970.

"Breve storia del romanzo poliziesco". Cruciverba. Torino: Einaudi, 1983. 216-31.

Zmegac, Victor. "Il paradosso storico-letterario del romanzo poliziesco". La trama del delitto: teoria e analisi del racconto poliziesco. Ed. Renzo Cremante e Loris Rambelli. Parma:

Pratiche Editrice, 1980. 183-93. 